\title{
Posttraumatic Stress Symptoms and the Diathesis-Stress Model of Chronic Pain and Disability in Patients Undergoing Major Surgery
}

\author{
Andrea L. Martin, MA,* Eileen Halket, RN, $\dagger$ Gordon J.G. Asmundson, PhD, $\dagger$ \\ David B. Flora, PhD,* and Joel Katz, PhD*†
}

\begin{abstract}
Objectives: To (1) use structural equation modeling (SEM) to examine relationships proposed in Turk's diathesis-stress model of chronic pain and disability as well as (2) investigate what role, if any, posttraumatic stress symptoms (PTSS) play in predicting pain disability, relative to some of the other factors in the model.

Methods: The study sample consisted of 208 patients scheduled for general surgery, 21 to 60 years of age (mean age $=47.18 \mathrm{y}$, $\mathrm{SD}=9.72 \mathrm{y}$ ), who reported experiencing persistent pain for an average of 5.56 years $(\mathrm{SD}=7.90 \mathrm{y})$. At their preadmission hospital visit, patients completed the Anxiety Sensitivity Index, Pain Catastrophizing Scale, Pain Anxiety Symptoms Scale-20, Pain Disability Index, posttraumatic stress disorder Checklist, and rated the average intensity of their pain ( 0 to 10 numeric rating scale). SEM was used to test a model of chronic pain disability and to explore potential relationships between PTSS and factors in the diathesis-stress model.
\end{abstract}

Results: SEM results provided support for a model in which anxiety sensitivity predicted fear of pain and catastrophizing, fear of pain predicted escape/avoidance, and escape/avoidance predicted pain disability. Results also provided support for a feedback loop between disability and fear of pain. SEM analyses provided preliminary support for the inclusion of PTSS in the diathesisstress model, with PTSS accounting for a significant proportion of the variance in pain disability.

Discussion: Results provide empirical support for aspects of Turk's diathesis-stress model in a sample of patients with persistent pain. Findings also offer preliminary support for the role of PTSS in fear-avoidance models of chronic pain.

Key Words: diathesis-stress, fear-avoidance, PTSS, chronic pain

T he role of psychologic and behavioral factors in the development and maintenance of chronic pain and disability has received increased attention in recent years. In particular, anxiety sensitivity, fear of pain, catastrophizing, and escape/avoidance behaviors are theorized to play a

Received for publication June 4, 2009; revised February 15, 2010; accepted February 17, 2010.

From the *Department of Psychology, York University; †Department of Anesthesia, The University of Toronto, Toronto, Ontario; and $\$$ Department of Psychology, University of Regina, Regina, Sakatchewan, Canada.

Supported by a CIHR Canada Research Chair in Health Psychology (J.K.); a CIHR Investigator Award and NET Grant (G.J.G.A.); as well as a CIHR Doctoral Award and a CIHR Strategic Training Fellowship in Pain: Molecules to Community (A.L.M.).

Reprints: Joel Katz, PhD, Department of Psychology, Behavioral Sciences Building, York University, 4700 Keele Street, Toronto, Ontario M3J 1P3, Canada (e-mail: ikatz@yorku.ca). critical role in the maintenance of many chronic pain conditions. ${ }^{1,2}$ In an effort to integrate these various factors and offer an explanation for individual differences in recovery after a trauma, Turk $^{3}$ proposed a diathesis-stress model of chronic pain and disability (Fig. 1). The model posits that individuals are more likely to develop avoidance responses and subsequent pain disability after a trauma if they are highly anxiety-sensitive, and thus experience increased body awareness and a fear of physiologic reactions; if they engage in catastrophic thinking involving interpreting pain sensations, and associated symptoms, as indicative of serious harm; and if they are predisposed to respond fearfully to pain.

Converging lines of evidence provide empirical support for various components of the diathesis-stress model. ${ }^{3}$ Clinical data indicate a link between anxiety sensitivity and chronic pain. Anxiety sensitivity is the fear of anxietyrelated sensations, such as a rapidly beating heart, based on the belief that these sensations will have harmful consequences. ${ }^{4}$ Highly anxiety-sensitive individuals experience increased body awareness and a fear of physiologic reactions, which in turn may make them more vigilant to pain-related sensations and more fearful of the noxious sensations associated with pain. ${ }^{5}$ High anxiety sensitivity is associated with more fearful appraisals of pain and greater pain-related escape/avoidance behaviors in individuals with chronic back pain, ${ }^{6}$ musculoskeletal pain, ${ }^{7}$ and recurrent headaches. ${ }^{8}$ Anxiety sensitivity has also been shown to predict fear of pain better than depression and pain severity in a heterogenous chronic pain population. ${ }^{9}$ Furthermore, in a sample of children and adolescents with chronic pain, anxiety sensitivity accounted for $38.6 \%$ of the variance in fear of pain and fear of pain accounted for $39.9 \%$ of the variance in pain-related disability. ${ }^{10}$

In addition to anxiety sensitivity, a number of studies have linked fear of pain to increased functional impairment and decreased levels of physical performance. ${ }^{11-14}$ Fear and avoidance behaviors have been linked to distress and disability in patients with headache, sickle cell disease, and musculoskeletal pain. ${ }^{1}$ Furthermore, fear of pain has been shown to predict disability and interference with activities of daily living better than anxiety, depression, and sensory pain. ${ }^{15}$

Research and clinical data also provide support for the role of catastrophizing in chronic pain. ${ }^{16}$ Similar to anxiety sensitivity, catastrophizing is thought to play a role in fearful and avoidant responses to pain through hypervigilance to threatening somatic cues and excessive focus on pain sensations. ${ }^{17}$ Pain catastrophizing is characterized by "... an exaggerated negative 'mental set' brought to bear during actual or anticipated pain experience" (pp. 53 in Ref. 18), unrealistic beliefs that the current situation will 


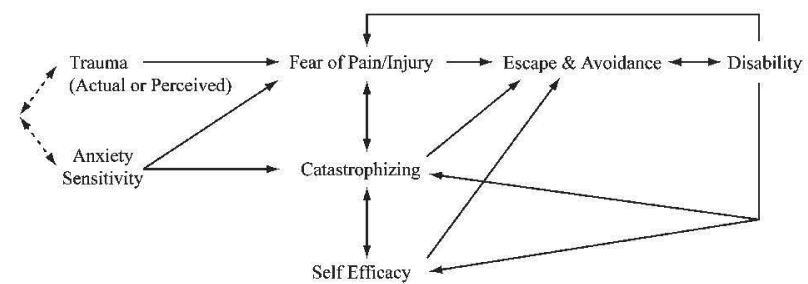

FIGURE 1. Turk's diathesis-stress model of chronic pain and disability. ${ }^{3}$ Reproduced with permission.

lead to the worst possible pain outcome, ${ }^{19}$ and negative thoughts about the future and self. ${ }^{3}$

Research demonstrates an association between catastrophizing and low back pain, ${ }^{20}$ mixed chronic pain, ${ }^{21}$ pain related to dental procedures, ${ }^{22}$ and acute postsurgical pain. ${ }^{23-25}$ Furthermore, Sullivan and colleagues ${ }^{17}$ found catastrophizing assessed 10 weeks before a painful procedure was predictive of subsequent pain ratings. Pain catastrophizing also predicts preoperative and postoperative pain ratings in patients undergoing total knee arthroplasty. ${ }^{26-28}$

Several fear-avoidance models have been proposed to integrate these various findings. On the basis of earlier studies, ${ }^{29-31}$ Vlaeyen and colleagues ${ }^{13}$ proposed a fearavoidance model of chronic pain in which catastrophic misinterpretations of pain set in motion a cycle of painrelated fear, escape/avoidance, and hypervigilence, leading to increased disability and impairment. ${ }^{2}$ Cook et $\mathrm{al}^{32}$ recently validated this model through the use of structural equation modeling (SEM). Asmundson and Taylor ${ }^{7}$ proposed a model in which anxiety sensitivity directly exacerbates fear of pain and indirectly increases painrelated escape/avoidance behaviors through its influence on fear of pain. They found that anxiety sensitivity accounted for $30 \%$ of the variance in fear of pain and fear of pain accounted for $68 \%$ of the variance in escape/avoidance behavior in adults with musculoskeletal injury. Norton and Asmundson $^{33}$ subsequently replicated this model in a sample of patients with recurrent headache pain.

Turk's ${ }^{3}$ diathesis-stress model expands on Asmundson and Taylor's ${ }^{7}$ model by including the influence of pain catastrophizing and self-efficacy. To date, the relationships among various components of the diathesis-stress model ${ }^{3}$ have been independently supported. However, additional research is required to simultaneously test the various links proposed in the diathesis-stress model to validate the model as a whole. Research is also needed to investigate the role of other factors that may broaden the diathesis-stress model. Specifically, the relationship between posttraumatic stress disorder (PTSD) and chronic pain is of growing interest. Research and clinical practice indicate these 2 disorders are highly comorbid. ${ }^{34-36}$

Two hypotheses, the shared vulnerability and the mutual maintenance hypotheses, have been proposed to account for the link between PTSD and chronic pain. The shared vulnerability hypothesis suggests that certain factors, namely anxiety sensitivity, may predispose individuals to the development of one or both of these conditions. ${ }^{34}$ Conversely, the mutual maintenance hypothesis suggests that certain components of each disorder may interact in such a way as to maintain or exacerbate the other. ${ }^{35}$ For example, for individuals with PTSD, chronic pain may serve as an ongoing reminder of the traumatic event, which in turn may lead to increased avoidance of pain sensations and ultimately increased levels of distress and disability. ${ }^{35}$ Given these observed links between PTSD and chronic pain, and the inclusion of trauma (perceived or actual) as a variable in the diathesis-stress model, ${ }^{3}$ the potential role of posttraumatic stress symptoms (PTSS) is of great interest.

This study had 2 aims: (1) to use SEM to test the relationships proposed in the diathesis-stress model of chronic pain and disability, ${ }^{3}$ and (2) to investigate the role, if any, played by PTSS in predicting pain disability, relative to other factors in the model. To address these aims, 2 statistical models were created and evaluated in 208 patients with persistent pain who were scheduled for major surgery. The first tested a Turk-based model of pain disability $^{3}$; and the second explored potential relationships between PTSS and the variables in the first model.

\section{MATERIALS AND METHODS}

\section{Participants}

The study sample consisted of 208 patients scheduled to undergo major surgery [abdominal $(68.72 \%)$, thoracic $(17.95 \%)$, and other $(13.33 \%) ; 124$ females and 83 males; 21 to 60 years of age (mean age $47.18 \mathrm{y}, \mathrm{SD} 9.72 \mathrm{y}$ )], who were experiencing persistent pain. Pain locations are presented in Table 1. Fifty-one percent of patients reported pain at more than one site. Of the 208 patients, 21 were unable to identify when their pain began. Four patients reported their pain began within the last year but were unable to identify the month of onset. The remaining 183 patients reported having experienced a persistent pain problem for an average of 5.56 years $(S D=7.90 \mathrm{y})$ and reported an average pain intensity of $5.70(\mathrm{SD}=2.45)$ on a 0 to 10 numeric rating scale (NRS).

The present sample was selected from a study database examining predictors of postoperative pain and the transition from acute to persistent pain after surgery. An earlier publication from this database examined the factor structure of PTSS. ${ }^{37}$ Patients were screened for preexisting pain conditions before their surgery and the present sample represents those individuals who reported a persistent pain problem. The nature of the pain was based on patient selfreport. Information regarding formal diagnoses and whether their surgery was related to their persistent pain condition is unknown.

\section{Measures and Procedure}

The research study was reviewed and approved by the Research Ethics Board at the Toronto General Hospital,

TABLE 1. Pain Locations Reported by Patients at Their Preadmission Hospital Visit

\begin{tabular}{lcc}
\hline Location of Pain & N & \% \\
\hline Abdomen & 60 & 28.85 \\
Back & 37 & 17.79 \\
Lower limb & 31 & 14.90 \\
Thorax & 26 & 12.50 \\
Pelvis & 20 & 9.62 \\
Head/neck & 17 & 8.17 \\
Upper limb & 9 & 4.33 \\
Whole body & 5 & 2.40 \\
Missing & 3 & 1.44 \\
Total & 208 & 100 \\
\hline
\end{tabular}


University Health Network, and by the Human Participants Review Committee at York University. Prospective patients were recruited at the preadmission hospital visit approximately 7 to 10 days before surgery. After informed written consent, patients were asked to rate the intensity of their current pain on a 0 to $10 \mathrm{NRS}$ and to complete the following questionnaires: the Anxiety Sensitivity Index (ASI) ${ }^{38}$ the Pain Catastrophizing Scale (PCS), ${ }^{17}$ the Pain Anxiety Symptoms Scale (PASS), ${ }^{39}$ the Pain Disability Index (PDI) ${ }^{40}$ and the PTSD Checklist - Civilian Version (PCL-C). ${ }^{41}$

\section{ASI}

The $\operatorname{ASI}^{38}$ is a widely used, 16 -item scale that measures concerns that anxiety and anxiety-related symptoms will lead to harmful negative consequences. Each item is rated on a 5 -point scale ranging from very little (0) to very much (4). The ASI yields a total score and 3 factor analytic derived subscale scores, including (1) fear of somatic symptoms/physical concerns, (2) fear of cognitive symptoms/mental incapacitation concerns, and (3) fear of publicly observable symptoms/social concerns. ${ }^{42}$ The ASI demonstrates good test-retest reliability $(r=0.72)$ and research demonstrates some evidence for the discriminant validity of the 3 subscales among anxiety disorder outpatients. $^{43}$

\section{PCS}

The $\mathrm{PCS}^{17}$ consists of 13 items describing thoughts and feelings that individuals may experience when they are in pain. Each item is rated on a 5-point scale ranging from not at all (0) to all the time (4). The PCS yields a total score and 3 subscale scores assessing (1) rumination, (2) magnification, and (3) helplessness. The PCS demonstrates adequate-to-excellent internal consistency in community $(\alpha=0.88$ to 0.95$)$ and pain outpatient samples $(\alpha=0.75$ to $0.92) .{ }^{44}$ Concurrent validity is also evidenced by a moderate correlation between the PCS and self-report measures of anxiety $(r=0.32)$ and negative affectivity $(r=0.70) .{ }^{45}$ The PCS shows good test-retest reliability at $6(r=0.75)$ and 10 weeks $(r=0.70){ }^{45}$

\section{PASS-20}

The PASS $-20^{39}$ is a shortened 20 -item version of the original PASS ${ }^{15}$ designed to measure fear and anxiety responses specific to pain. The PASS-20 has four 5-item subscales, including (1) cognitive anxiety, (2) escape and avoidance, (3) fearful thinking, and (4) physiologic anxiety. Each item is rated on a 6-point scale ranging from never $(0)$ to always (5), with total scores ranging from 0 to 100 . The PASS-20 has been shown to have good internal consistency $(\alpha=0.81$ ), and good convergent validity with the original 40 -item PASS $(r=0.95) .39$ Concurrent validity of the PASS-20 is demonstrated through its moderate-to-high correlations with related measures such as ASI $(r=0.56)$, fear of pain (fear of pain questionnaire-III; $r=0.53$ ), and $\operatorname{PCS}(r=0.38){ }^{46}$

\section{PDI}

The $\mathrm{PDI}^{40}$ assesses the extent to which persistent pain interferes with an individual's ability to engage in 7 different areas of everyday activity including: (1) family/ home responsibilities, (2) recreation, (3) social activity, (4) occupation, (5) sexual behavior, (6) self-care, and (7) lifesupport activity. Each item is rated on an 11-point scale ranging from no disability $(0)$ to total disability $(10)$. The PDI demonstrates good construct validity as evidenced by its significant relationship with other measures of painrelated disability and distress. ${ }^{47,48}$ Test-retest reliability of the PDI is good $(r=0.91)^{49}$ and the internal consistency is high $(\alpha=0.86) .{ }^{50}$

\section{PCL-C}

The PCL-C ${ }^{41}$ is a 17 -item self-report measure. Each item is a statement based on the current Diagnostic and Statistical Manual of Mental Disorder-IV symptoms for PTSD. Respondents are asked to indicate how much they have been bothered by each symptom over the past month on a 5-point scale ranging from not at all (1) to extremely (5). The questionnaire produces a total score and 4 subscale scores, ${ }^{51}$ including (1) re-experiencing, (2) avoidance, (3) numbing, and (4) hyperarousal. Test-retest reliability for the PCL-C over a 2 to 3 day retest interval is $0.96 .{ }^{52}$ Cut-off scores of $44^{52}$ and $50^{53}$ have been shown to reliably predict PTSD diagnosis.

\section{NRS for Pain Intensity}

Pain intensity was measured using a self-report NRS $^{54}$ ranging from 0 to 10 , with endpoints representing no pain $(0)$ and the most intense pain imaginable (10). Patients were asked to choose the number that best corresponds to the average intensity of their pain. The NRS provides a simple, efficient, and minimally intrusive measure of pain intensity. This scale is commonly used in clinical settings ${ }^{55}$ and is the preferred pain rating scale among patients. ${ }^{56}$ The NRS is highly correlated $(r=0.94)$ with the Visual Analog Scale ${ }^{55}$ and is sensitive to change following pharmacologic interventions. ${ }^{54}$

\section{Data Analysis}

The first objective of this study was to use SEM to allow a comprehensive investigation of the relationships proposed in the diathesis-stress model of chronic pain and disability. ${ }^{3}$ The hypothesized model (Fig. 2) predicts a direct effect of anxiety sensitivity on fear of pain (path 1), anxiety sensitivity on catastrophizing (path 2), fear of pain on escape/avoidance behaviors (path 3), catastrophizing on escape/avoidance behaviors (path 4), and escape/avoidance behaviors on pain disability (path 5). Furthermore, this model predicts that pain intensity will have a direct effect on fear of pain (path 6), catastrophizing (path 7), escape/ avoidance (path 8), and disability (path 9). Pain intensity was included in the model to make explicit the contribution of pain to disability and because it is the main factor prompting patients to seek help. Two of the feedback loops proposed in the model $^{3}$ were also tested; these include a direct effect of disability on fear of pain (path 10) and

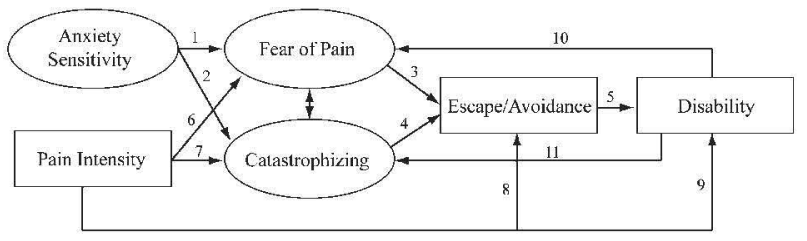

FIGURE 2. Path diagram of hypothesized relationships in Turk diathesis-stress model of chronic pain and disability. ${ }^{3}$ 
disability on catastrophizing (path 11). Self-efficacy was not evaluated in this adaptation of the diathesis-stress model. ${ }^{3}$

In the model in Figure 2 and in subsequent figures, anxiety sensitivity, fear of pain, and catastrophizing are depicted as latent variables (using ovals) rather than observed variables (using rectangles) because multiple indicators, namely the subscale scores from each measure, were available for these constructs. Using latent variables for constructs leads to path estimates that are unbiased by measurement error. This strategy was based on results of Coffman and MacCallum, ${ }^{57}$ who found that fitting models using item parcels (ie, the subscale scores) as latent variable indicators is superior to using path analyses with observed total score indicators adjusted by estimated reliability.

The second objective of this study was to investigate what role, if any, PTSS play in predicting pain disability, relative to other factors in the diathesis-stress model. ${ }^{3}$ Although this study did not use a psychiatric population, community-based studies indicate that the lifetime prevalence for experiencing a traumatic event is $89.6 \%^{58}$ and the lifetime prevalence for PTSD is approximately $8 \% .{ }^{59}$ Furthermore, many more individuals not meeting diagnostic criteria for a full diagnosis of PTSD may experience partial or sub-threshold PTSS. ${ }^{60} \mathrm{We}$, therefore, anticipated a range of severity in PTSS within the present sample and found $22.7 \%$ of PCL-C scores fell within the clinical range (Fig. 3)

To address the second objective of this study, a second model (Fig. 4) was developed based on the shared vulnerability and mutual maintenance hypotheses linking PTSD and chronic pain. As described earlier, the shared vulnerability hypothesis proposes that anxiety sensitivity may predispose individuals to the development of both PTSD and chronic pain. ${ }^{34}$ As such, a direct path from anxiety sensitivity to PTSS was hypothesized. Furthermore, the mutual maintenance hypothesis suggests that certain components of chronic pain may maintain symptoms of PTSD and vice versa. ${ }^{35}$ Accordingly, pain intensity was hypothesized to have a direct relationship with PTSS and PTSS were hypothesized to have a direct effect on escape/ avoidance behavior. Bidirectional relationships among fear of pain, catastrophizing, and PTSS were also proposed.

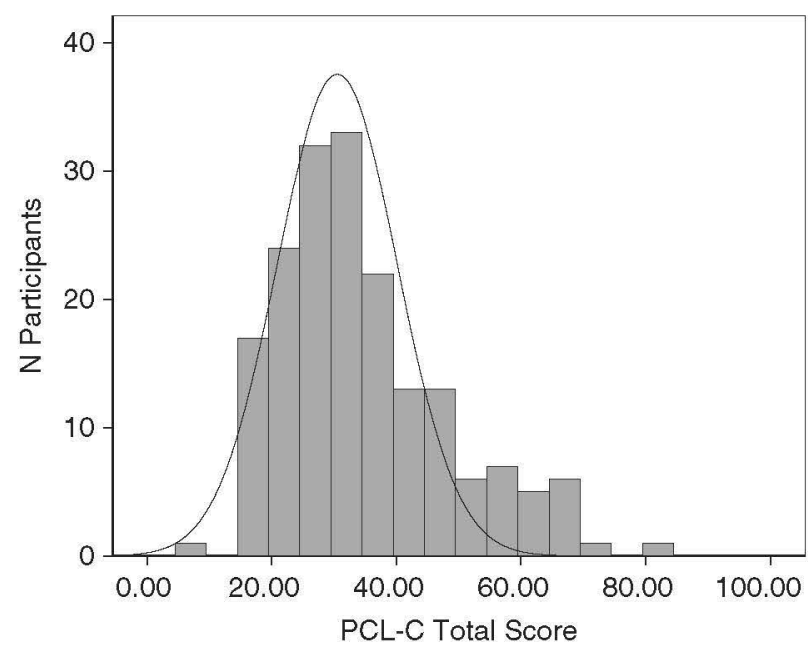

FIGURE 3. Distribution of posttraumatic stress disorder Checklist-Civilian Version scores across participants.

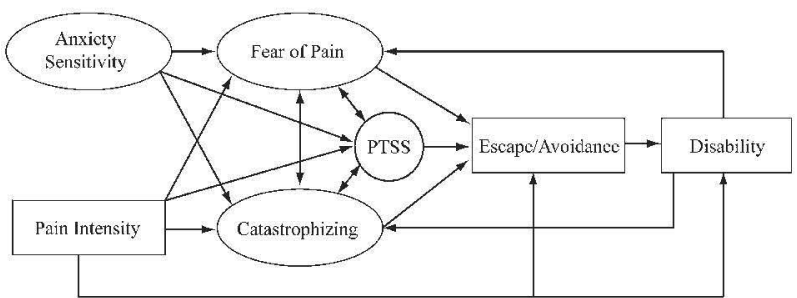

FIGURE 4. Path diagram of hypothesized relationships in a modified diathesis-stress mode $^{3}$ that includes posttraumatic stress symptoms.

Before estimating the models, the manifest variables were examined for univariate and multivariate outliers and distribution. Table 2 presents descriptive statistics for each of the manifest variables. SEM was performed according to a 2-stage process using AMOS 7.0 (SPSS Inc, Chicago, IL). First, separate confirmatory factor analysis models were used to verify that the subscale scores used to measure each of the latent variables formed a single factor. To include cases with missing values, a one-factor model was fitted to the observed covariance matrix using full information maximum likelihood estimation ${ }^{61}$ and fit statistics were examined to confirm that a one-factor model was a good fit to the data for a given set of subscales.

After verifying the factor structure of each of the latent variables, the hypothesized models in Figures 2 and 3 were estimated, again using full information maximum likelihood. In addition to $\chi^{2}$ goodness-of-fit tests, 5 fit statistics were computed for each model: root-mean-square error of approximation (RMSEA), comparative fit index (CFI), Tucker-Lewis index (TLI), Akaike information criterion (AIC), and Browne-Cudeck criterion (BCC). The RMSEA reflects the magnitude of difference between the fitted and actual covariance matrices with a parsimony correction for number of parameters. Values of RMSEA $\leq 0.05$ indicate close fit and RMSEA $0.05 \leq 0.08$ suggests a reasonable model fit. ${ }^{62}$ The CFI assesses the relative improvement in fit of the proposed model compared with a null model with no specified relations. ${ }^{62}$ Values of CFI $\geq 0.90$ indicate reasonable model fit. The TLI assesses the relative improvement per degree of freedom of the target model over the null model. ${ }^{63}$ Similar to the CFI, values of TLI $\geq 0.90$ indicate reasonable model fit. Finally, the AIC assesses model fit in hypothetical replication samples of the same size and randomly drawn from the same population as the research sample. ${ }^{62}$ The AIC is generally used to select among nonnested competing models estimated with the same data. The $\mathrm{BCC}^{64}$ operates in the same manner as the $\mathrm{AIC}$ but imposes greater penalties. ${ }^{65}$ The model with the smallest AIC and $\mathrm{BCC}$ is considered to have the best fit.

\section{RESULTS}

\section{Evaluation of a Diathesis-Stress Model of Chronic Pain Disability}

Means and SDs for each of the measures used in the analyses are presented in Table 2 and correlation coefficients among model variables are presented in Table 3 . On the basis of the proposed diathesis-stress model, ${ }^{3}$ a model with 3 manifest and 3 latent variables was developed (Model 1, Fig. 5). As illustrated in Figure 5, the 3 subscales of the ASI: ASI-fear of somatic symptoms/physical 
TABLE 2. Descriptive Statistics of Measures Used in Structural Equation Modeling ( $N=208)$

\begin{tabular}{|c|c|c|c|c|}
\hline Measure & Mean & SD & $\alpha$ & $\%$ Missing \\
\hline Pain intensity & 5.70 & 2.45 & - & 6.70 \\
\hline \multicolumn{5}{|l|}{ ASI } \\
\hline Fear of somatic symptoms & 11.61 & 7.32 & 0.89 & 12.00 \\
\hline Fear of cognitive symptoms/mental incapacitation & 2.62 & 3.18 & 0.86 & 12.50 \\
\hline Fear of publicly observable symptoms/social concerns & 5.52 & 2.37 & 0.50 & 12.00 \\
\hline \multicolumn{5}{|l|}{ PCS } \\
\hline Rumination & 7.68 & 4.55 & 0.91 & 11.50 \\
\hline Magnification & 3.81 & 2.74 & 0.73 & 11.50 \\
\hline Helplessness & 7.84 & 5.78 & 0.91 & 11.10 \\
\hline \multicolumn{5}{|l|}{ PASS-20 } \\
\hline Physiologic anxiety & 7.71 & 6.35 & 0.84 & 12.50 \\
\hline Cognitive anxiety & 12.57 & 6.25 & 0.83 & 12.50 \\
\hline Fearful thinking & 8.77 & 6.73 & 0.82 & 12.50 \\
\hline Escape and avoidance & 11.85 & 6.12 & 0.77 & 12.50 \\
\hline \multicolumn{5}{|l|}{ PCL-C } \\
\hline Re-experiencing & 9.45 & 4.60 & 0.89 & 13.00 \\
\hline Avoidance & 3.89 & 2.02 & 0.73 & 13.50 \\
\hline Emotional numbing & 10.17 & 4.48 & 0.83 & 13.50 \\
\hline Hyperarousal & 11.38 & 4.49 & 0.81 & 13.50 \\
\hline PDI & 27.19 & 18.87 & - & 11.50 \\
\hline
\end{tabular}

ASI indicates Anxiety Sensitivity Index; PASS-20, Pain Anxiety Symptoms Scale-20; PCL-C, Posttraumatic Stress Disorder Checklist-Civilian Version; PCS, Pain Catastrophizing Scale; PDI, Pain Disability Index.

concern, ASI - cognitive symptoms/mental incapacitation concerns, and ASI - publicly observable symptoms/social concerns, formed an anxiety sensitivity latent variable; the 3 subscales of the PASS-20: PASS - cognitive anxiety, PASS - fearful thinking, and PASS - physiologic anxiety, formed a fear of pain latent variable; and finally the 3 subscales of the PCS: PCS - rumination, PCS - magnification, and PCS - helplessness, formed a pain catastrophizing latent variable. Pain intensity, escape/avoidance, and pain disability were estimated by the NRS, PASS-Escape/ Avoidance subscale, and PDI total score, respectively.
Model fit statistics indicated adequate fit of the hypothesized model to the data (RMSEA $=0.07 ; \mathrm{CFI}=$ 0.97 ; and TLI $=0.94)$. The $\chi^{2}$ statistic was significant $\left[\chi^{2}\right.$ $(45, \mathrm{~N}=208)=87.15, P<0.05]$; however, this statistic is generally not regarded as an ideal fit statistic as it is highly sensitive to sample size and the size of correlations ${ }^{62}$ and tests the unrealistic null hypothesis of perfect fit. ${ }^{66}$ Therefore, results provide support for the hypothesized model. As expected, each manifest variable had a salient loading on its specified latent variable. All of the standardized path coefficients shown in Figure 5 were significant with the

TABLE 3. Zero-order Correlations Among Variables in Structural Model

\begin{tabular}{|c|c|c|c|c|c|c|c|c|c|c|c|c|c|c|c|c|}
\hline & 1 & 2 & 3 & 4 & 5 & 6 & 7 & 8 & 9 & 10 & 11 & 12 & 13 & 14 & 15 & 16 \\
\hline 1. Pain & 1 & 0.08 & 0.10 & 0.06 & $0.28 * *$ & $0.18 *$ & $0.18^{*}$ & $0.20^{*}$ & $0.20 * *$ & $0.15^{*}$ & $0.24 * *$ & $0.25^{* * *}$ & $0.25^{* * *}$ & $0.18 *$ & $0.21 * *$ & $0.29 * *$ \\
\hline 2. ASI-SOM & - & 1 & $0.68 * *$ & $0.43 * *$ & $0.49 * *$ & $0.59 * *$ & $0.54 * *$ & $0.54 * *$ & $0.61 * *$ & $0.68 * *$ & $0.44 * *$ & $0.32 * *$ & $0.32 * *$ & $0.38 * *$ & $0.44 * *$ & $0.22 * *$ \\
\hline 3. ASI-COG & - & - & 1 & $0.47 * *$ & $0.38 * *$ & $0.56^{* *}$ & $0.55^{* *}$ & $0.48 * *$ & $0.49 * *$ & $0.60 * *$ & $0.29 * *$ & $0.34 * *$ & $0.31 * *$ & $0.33^{*}$ & $0.40 * *$ & $0.25 * *$ \\
\hline 4. ASI-PUB & - & - & - & 1 & $0.34^{* *}$ & $0.43^{\text {** }}$ & $0.39 * *$ & $0.32^{* * *}$ & $0.34 * *$ & $0.36^{* *}$ & $0.22 * *$ & $0.18^{*}$ & $0.21^{* * *}$ & $0.25^{* *}$ & $0.25 * *$ & $0.17^{*}$ \\
\hline 5. PCS-RUM & - & - & — & - & 1 & $0.70 * *$ & $0.71 * *$ & $0.54 * *$ & $0.62 * *$ & $0.60 * *$ & $0.54 * *$ & $0.39 * *$ & $0.40 * *$ & $0.40 * *$ & $0.37 * *$ & $0.32 * *$ \\
\hline 6. PCS-MAG & - & - & - & - & - & 1 & $0.73^{* *}$ & $0.64^{* * *}$ & $0.56 * *$ & $0.71 * *$ & $0.49 * *$ & $0.43^{* *}$ & $0.40^{* * *}$ & $0.43^{* *}$ & $0.36 * *$ & $0.31 * *$ \\
\hline 7. PCS-HELP & - & - & - & - & — & - & 1 & $0.59 * *$ & $0.58 * *$ & $0.63^{* *}$ & $0.48 * *$ & $0.33^{* *}$ & $0.34^{* *}$ & $0.35^{* *}$ & $0.41 * *$ & $0.33^{* *}$ \\
\hline 8. PASS-PA & - & - & - & — & — & - & - & 1 & $0.63 * *$ & $0.76^{* *}$ & $0.61 * *$ & $0.43 * *$ & $0.46^{* *}$ & $0.35 * *$ & $0.36 * *$ & $0.41 * *$ \\
\hline 9. PASS-CA & - & - & - & - & - & - & - & - & 1 & $0.70^{* *}$ & $0.62 * *$ & 0.41 ** & $0.43^{* *}$ & $0.41 * *$ & $0.44 * *$ & $0.43^{* *}$ \\
\hline 10. PASS-FT & - & - & - & - & - & - & - & - & - & 1 & $0.59 * *$ & $0.40 * *$ & $0.46^{* *}$ & $0.41 * *$ & $0.39 * *$ & $0.40 * *$ \\
\hline 11. PASS-E/A & 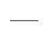 & - & - & - & - & - & - & - & - & - & - & $0.28 * *$ & $0.34 * *$ & $0.27 * *$ & $0.25 * *$ & $0.47 * *$ \\
\hline 12. PCL-RE-EXP & - & - & - & - & - & - & - & - & - & - & - & 1 & $0.70^{* * *}$ & $0.62 * *$ & $0.58 * *$ & $0.29 * *$ \\
\hline 13. PCL-AVOID & - & - & - & - & - & - & - & - & - & - & - & - & 1 & $0.69 * *$ & $0.54 * *$ & $0.35 * *$ \\
\hline 14. PCL-NUMB & - & - & - & - & - & - & - & - & - & - & - & - & - & 1 & $0.70 * *$ & $0.46^{* *}$ \\
\hline 15. PCL-AROUS & - & - & - & - & - & - & - & - & - & - & - & - & - & - & 1 & $0.39 * *$ \\
\hline 16. PDI & - & - & - & - & - & - & - & - & - & - & - & - & - & - & - & 1 \\
\hline
\end{tabular}

$* P<0.05$.

$* * P<0.01$.

ASI indicates Anxiety Sensitivity Index; ASI-COG, ASI-fear of cognitive symptoms/mental incapacitation concerns; ASI-PUB, ASI-fear of publicly observable symptoms/social concerns; ASI-SOM, ASI somatic symptoms/physical concerns; PASS, Pain Anxiety Symptoms Scale; PASS-CA, PASScognitive anxiety; PASS-E/A, PASS - escape/avoidance; PASS-FT, PASS - fearful thinking; PASS-PA, PASS-physiological anxiety; PCL, PTSD Checklist; PCL-AROUS, PCL-hyperarousal; PCL-AVOID, PCL-avoidance; PCL-NUMB, PCL-numbing; PCL-RE-EXP, PCL-re-experiencing; PCS, Pain Catastrophizing Scale; PCS-HELP, PCS helplessness; PCS-MAG, PCS - magnification; PCS-RUM, PCS rumination; PDI, Pain Disability Index total score. 


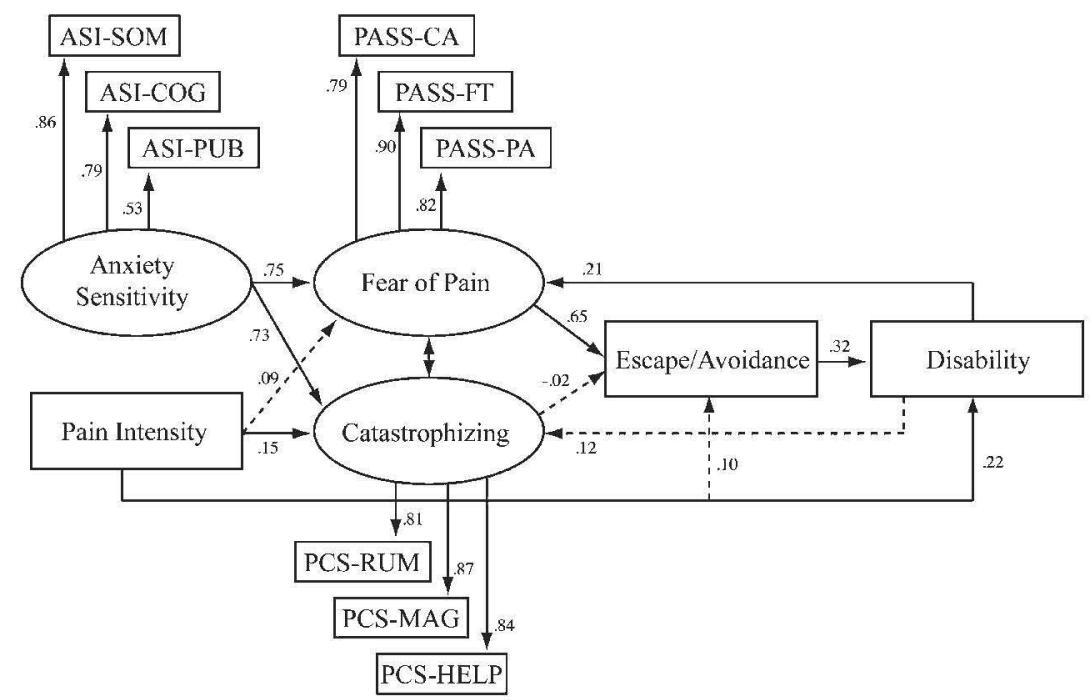

FIGURE 5. Structural equation Model 1, based on the diathesis-stress model of chronic pain and disability. ${ }^{3}$ Standardized path coefficients are presented. ASI indicates Anxiety Sensitivity Index; ASI-COG, ASI_fear of cognitive symptoms/mental incapacitation concerns; ASI-PUB, ASI-fear of publicly observable symptoms/social concerns; ASI-SOM, ASI-somatic symptoms/physical concerns; Disability, Pain Disability Index total score; Escape/Avoidance, PASS-20_escape/avoidance; PASS, Pain Anxiety Symptoms Scale; PASSCA, PASS-20 - cognitive anxiety; PASS-FT, PASS-20 - fearful thinking; PASS-PA, PASS-20 - physiologic anxiety; PCS, Pain Catastrophizing Scale; PCS-HELP, PCS-helplessness; PCS-MAG, PCS-magnification; PCS-RUM, PCS—rumination. Dotted lines represent nonsignificant paths $(P>0.05)$.

exception of the paths represented by dotted lines. Specifically, the paths from pain intensity to fear of pain $(P=0.15)$, pain intensity to escape/avoidance $(P=0.09)$, catastrophizing to escape/avoidance $(P=0.89)$, and pain disability to catastrophizing $(P=0.08)$ were not significant.

Interpretation of SEM analyses is strengthened by comparison with alternative models. ${ }^{67}$ Thus, a modified model was tested in which pain intensity and anxiety sensitivity were hypothesized to influence pain catastrophizing directly, which in turn was hypothesized to have a direct influence on fear of pain (Model 2, Fig. 6). Furthermore, a feedback loop from disability to pain intensity was also proposed. These hypothesized alternate relationships were based on the Vlaeyen-Linton fearavoidance model ${ }^{2}$ and subsequent modifications to this model by Norton and Asmundson, ${ }^{68}$ who proposed a direct effect of anxiety sensitivity on pain catastrophizing. Although the $\chi^{2}$ test was significant $\left[\chi^{2}(51, N=208)=\right.$ 103.64, $P<0.05]$, other model fit indices indicate an adequate fit of Model 2 to the data (RMSEA =0.07; CFI $=0.96$; and TLI $=0.93$ ). Furthermore, as illustrated in Figure 6, all standardized path coefficients were significant. The AIC and $\mathrm{BCC}$ values for Model $1(\mathrm{AIC}=177.15$ and $\mathrm{BCC}=183.18$ ) were smaller than those for Model 2

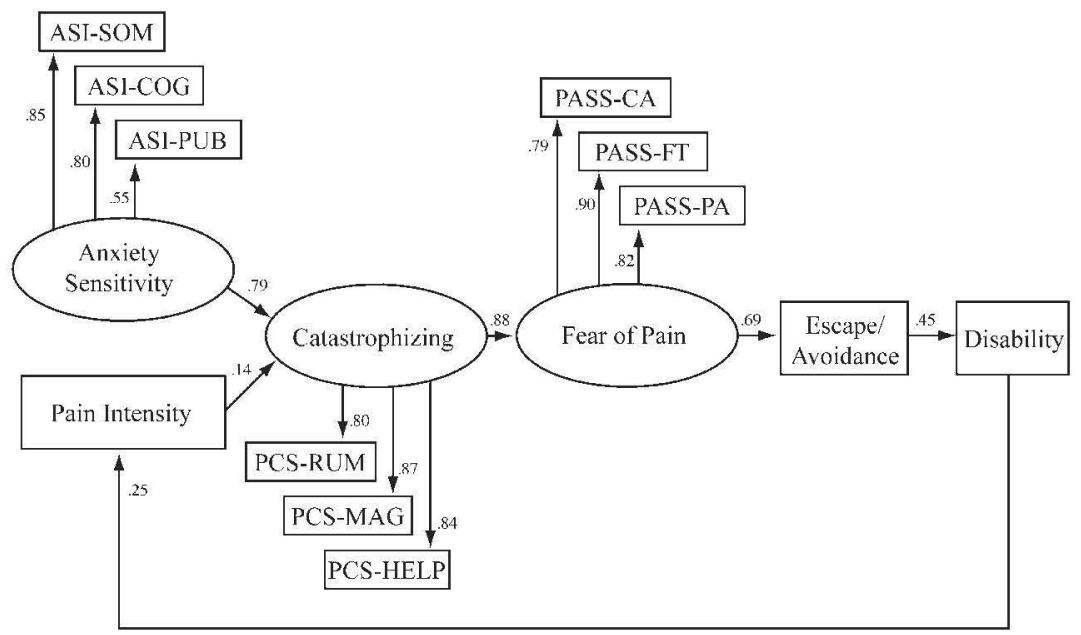

FIGURE 6. Structural equation Model 2. Standardized path coefficients are presented. ASI indicates Anxiety Sensitivity Index; ASI-COG, ASI_fear of cognitive symptoms/mental incapacitation concerns; ASI-PUB, ASI_fear of publicly observable symptoms/social concerns; ASI-SOM, ASI—-somatic symptoms/physical concerns; Disability, Pain Disability Index total score; Escape/Avoidance, PASS-20_escape/ avoidance; PASS, Pain Anxiety Symptoms Scale; PASS-CA, PASS-20_cognitive anxiety; PASS-FT, PASS-20_fearful thinking; PASS-PA, PASS-20_physiological anxiety; PCS, Pain Catastrophizing Scale; PCS-HELP, PCS - helplessness; PCS-MAG, PCS - magnification; PCSRUM, PCS-rumination. Dotted lines represent nonsignificant paths $(P>0.05)$. 
$(\mathrm{AIC}=181.64$ and $\mathrm{BCC}=186.86)$, suggesting the Turkbased Model 1 offers a closer fit to the data.

\section{Role of PTSS in Predicting Pain Disability}

A second set of SEM analyses were computed to investigate what role, if any, PTSS play in predicting pain disability in conjunction with the other factors in the diathesis-stress model. ${ }^{3}$ Specifically, the hypothesized structural model predicts a direct effect of anxiety sensitivity and pain intensity on PTSS and a direct effect of PTSS on escape/avoidance behaviors (Model 3, Fig. 7). Bidirectional relationships between PTSS, fear of pain, and catastrophizing were also proposed. Although the $\chi^{2}$ test was significant $\left[\chi^{2}(90, N=208)=185.00, P<0.05\right]$, other model fit indices indicate close fit of the model to the data $(\mathrm{RMSEA}=0.07 ; \mathrm{CFI}=0.94$; and $\mathrm{TLI}=0.91)$. The standardized path coefficients shown in Figure 8 were significant with the exception of the paths represented by dotted lines. Specifically, the paths from pain intensity to fear of pain $(P=0.07)$, pain intensity to escape/avoidance $(P=0.06)$, catastrophizing to escape/avoidance $(P=0.98)$, PTSD symptoms to escape/avoidance $(P=0.23)$, disability to fear of pain $(P=0.12)$, and disability to catastrophizing $(P=0.65)$ were not significant.

An alternate competing model was also tested based on recent findings that PTSS and pain intensity independently predict pain disability ${ }^{69}$ and the possibility that high levels of fear, avoidance, and catastrophizing may be indicative of a traumatic stress reaction to pain (involving symptoms of intrusive thoughts, emotional numbing, behavioral avoidance, and hyperarousal), which in turn may relate to increased pain disability. As such, an alternate model was proposed in which fear of pain, catastrophizing, and avoidance behaviors were hypothesized to directly influence PTSS, which in turn were hypothesized to directly influence pain disability (Model 4, Fig. 8). Goodness-of-fit indices indicate close fit of Model 4 to the data (RMSEA $=0.07$; $\mathrm{CFI}=0.95$; and TLI $=0.92$ ), again with the exception of the $\chi^{2}$ statistic, $\left[\chi^{2}(92, N=208)=181.68, P<0.05\right]$. Standardized path coefficients for this model are shown in Figure 8. All paths were significant with the exception of the paths from escape/avoidance to PTSS $(P=0.18)$ and catastrophizing to PTSS $(P=0.41)$. The AIC and BCC values for Model $4(\mathrm{AIC}=301.68$ and $\mathrm{BCC}=312.42$ ) were lower than those for Model $3(\mathrm{AIC}=309$ and $\mathrm{BCC}=320.10)$, suggesting Model 4 offers a better fit to these data.

\section{DISCUSSION}

The results of this study provide preliminary support for the diathesis-stress model of chronic pain proposed by Turk. ${ }^{3}$ The first model we examined (Model 1, Fig. 5), identified anxiety sensitivity as having significant direct effects on fear of pain and catastrophizing, accounting for $75 \%$ and $73 \%$ of the variance, respectively. Furthermore, fear of pain had a direct effect on escape/avoidance, accounting for $65 \%$ of the variance, and escape/avoidance had a direct effect on pain disability, accounting for $32 \%$ of the variance. Preliminary evidence for a potential feedback loop proposed in the diathesis-stress model of chronic pain ${ }^{3}$ was also evident as pain disability had a direct effect on fear of pain, accounting for $21 \%$ of the variance.

However, pain catastrophizing did not have a significant effect on escape/avoidance and disability did not

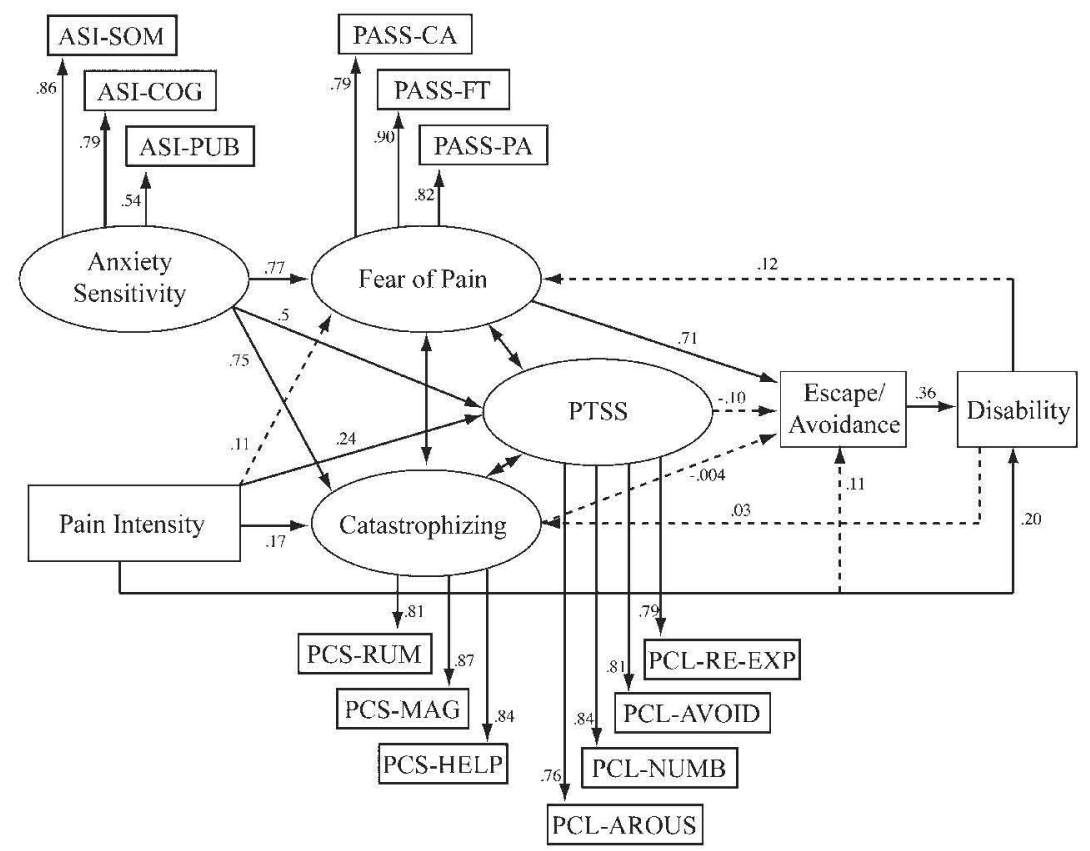

FIGURE 7. Structural equation Model 3 , based on a modified diathesis-stress model ${ }^{3}$ incorporating posttraumatic stress symptoms. Standardized path coefficients are presented. ASI indicates Anxiety Sensitivity Index; ASI-COG, ASI—fear of cognitive symptoms/mental incapacitation concerns; ASI-PUB, ASI-fear of publicly observable symptoms/social concerns; ASI-SOM, ASI—somatic symptoms/ physical concerns; Disability, Pain Disability Index total score; Escape/Avoidance, PASS-20_escape/avoidance; PASS, Pain Anxiety Symptoms Scale; PASS-CA, PASS-20-Cognitive anxiety; PASS-FT, PASS-20_fearful thinking; PASS-PA, PASS-20_physiological anxiety; PCL, PTSD Checklist; PCL-AROUS, PCL-hyperarousal; PCL-AVOID, PCL_avoidance; PCL-NUMB, PCL_numbing; PCL-RE-EXP, PCLre-experiencing; PCS, Pain Catastrophizing Scale; PCS-HELP, PCS-helplessness; PCS-MAG, PCS-magnification; PCS-RUM, PCSrumination; PTSS, posttraumatic stress symptoms. Dotted lines represent nonsignificant paths $(P>0.05)$. 


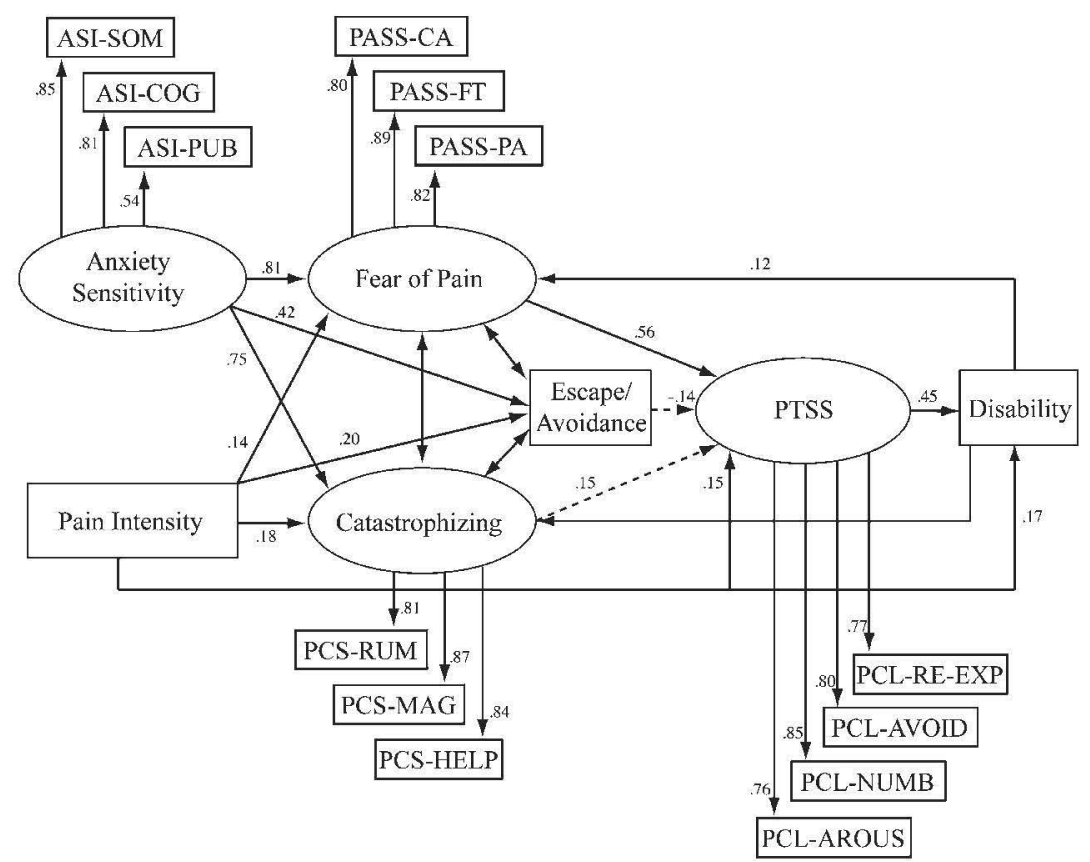

FIGURE 8. Structural equation Model 4. Standardized path coefficients are presented. ASI indicates Anxiety Sensitivity Index; ASI-COG, ASI_fear of cognitive symptoms/mental incapacitation concerns; ASI-PUB, ASI_fear of publicly observable symptoms/social concerns; ASI-SOM, ASI_-somatic symptoms/physical concerns; Disability, Pain Disability Index total score; Escape/Avoidance, PASS-20_escape/ avoidance; PASS, Pain Anxiety Symptoms Scale; PASS-CA, PASS-20—cognitive anxiety; PASS-FT, PASS-20-fearful thinking; PASS-PA, PASS-20_physiological anxiety; PCL, PTSD Checklist; PCL-AROUS, PCL-hyperarousal; PCL-AVOID, PCL-avoidance; PCL-NUMB, PCL_numbing; PCL-RE-EXP, PCL_re-experiencing; PCS, Pain Catastrophizing Scale; PCS-HELP, PCS—helplessness; PCS-MAG, PCS— magnification; PCS-RUM, PCS—rumination; PTSD, posttraumatic stress disorder. Dotted lines represent nonsignificant paths $(P>0.05)$.

have a significant direct effect on pain catastrophizing. Although the hypothesized direct effect of catastrophizing on escape/avoidance was not significant, it is still possible that catastrophizing had an indirect effect on escape/ avoidance through its relationship with fear of pain. Further research is needed to determine the extent to which pain catastrophizing plays a causal role in pain disability as predicted by the diathesis-stress model. ${ }^{13}$

The results also indicate that pain intensity had a significant direct effect on pain catastrophizing and pain disability. However, contrary to expectation, pain intensity did not have a significant direct effect on fear of pain or escape/avoidance behaviors. Earlier research also indicated that the direct effect of pain severity on escape/avoidance is small among patients with recurrent headache pain. ${ }^{33}$

This study also sought to examine the potential role of PTSS in predicting pain-disability in relation to some of the other factors in the diathesis-stress model. ${ }^{3}$ Results provided preliminary support for a model in which anxiety sensitivity and pain intensity had a direct effect on fear of pain, fear of pain and pain intensity had direct effects on PTSS, and PTSS and pain intensity had direct effects on pain disability (Model 4, Fig. 8). Pain intensity accounted for $15 \%$ of the variance in PTSS, providing further support for a relationship between PTSS and persistent pain. Pain intensity also had a direct effect on pain disability, accounting for $17 \%$ of the variance; however, PTSS had an even larger effect on pain disability, accounting for $45 \%$ of the variance.

Model 4 allowed for correlations among fear of pain, catastrophizing, and escape/avoidance and predicted that each in turn would have a direct effect on PTSS. Interestingly, only fear of pain had a significant direct effect on PTSS, accounting for $56 \%$ of the variance. However, although catastrophizing and escape/avoidance behaviors did not have direct effects on PTSS, this does not rule out possible indirect effects of these variables on PTSS through their relationship with fear of pain. That is, high levels of catastrophizing and escape/avoidance behaviors may increase fear of pain, which in turn exacerbates PTSS.

Although analyses suggest that Models 1 and 4 have an adequate fit to the data, it is important to note that these findings do not rule out the possibility of equivalent, or superior, alternate models not tested in this study. Furthermore, the differences in AIC and BCC values between each of the competing models were small, indicating a minimal improvement in fit for Model 1 relative to Model 2 and for Model 4 relative to Model 3. Replication in a different sample is advised before conclusive statements can be made regarding the superiority of one model over the others.

It is also important to note these findings do not imply causality; the data were cross-sectional and as such direction of influence cannot be confirmed. It is therefore difficult to determine how and why PTSS may be related to the variables in the models we examined (Figs. 6 and 7). One potential hypothesis, consistent with Model 4 (Fig. 8), is that individuals with persistent pain who experience a dysfunctional fear-avoidance cycle may be more vulnerable or less able to cope with stressful life events, thus making them more susceptible to the development of PTSS. ${ }^{70}$ This explanation leaves open the possibility that PTSS may develop in relation to an initiating painful event.

There are several limitations to this study. First, although numerous patients endorsed the presence of 
PTSS, the exact nature of the traumatic event to which patients were referring is unknown. Therefore, although a significant positive relationship between PTSS and pain disability was observed, the extent to which PTSS were related to a painful traumatic event is indeterminate. In addition, information pertaining to the lifetime or current psychologic histories of these patients is unknown and may provide valuable insight into the relationships among the variables in the proposed models. It is possible that some individuals were experiencing PTSS in response to the event that caused their chronic pain, whereas others were not. This is an important distinction that has yet to be addressed in the literature. ${ }^{34,71}$ In addition, the PTSS reported by some patients may have been exacerbated in response to a major medical diagnosis and their upcoming surgery. The present data offer a preliminary investigation into the potential relationships among PTSS and the variables in the diathesis-stress model of chronic pain and disability ${ }^{3}$; future study in surgical patients and in other populations is needed to replicate the present findings.

Furthermore, these data were collected at one point in time and experimental manipulations were not applied; therefore, as mentioned earlier, the direction of causality is unknown. Finding a model that fits the data closely does not rule out the possibility of other potential causal models. The small ratio of participants to observed variables is also important to note, as covariances become less stable when estimated from small sample sizes. ${ }^{72}$ Future research is needed to examine these models prospectively in larger samples of patients to better understand the nature of vulnerability and the direction of causality. Finally, an important omission from our model was the role of selfefficacy, which is included in the original model. ${ }^{3}$ Future research should continue to investigate the relationships proposed in the diathesis-stress model, particularly with respect to the role of self-efficacy and the addition of PTSS.

In conclusion, these results provide empirical support for aspects of Turk's diathesis stress $\operatorname{model}^{3}$ in a heterogeneous sample of patients with persistent pain. These findings also offer preliminary support for the potential role of PTSS in fear-avoidance models of chronic pain, thus elaborating the complex inter-relationships proposed in the diathesis stress model $^{3}$ and adding to the growing evidencebase for a relationship between PTSD and chronic pain. Clinicians treating individuals with pain or PTSS should be aware of the frequent concurrence of these 2 conditions as modifying treatment protocols to address and manage symptoms of both conditions will likely improve the treatment outcomes.

\section{ACKNOWLEDGMENT}

The authors thank Maria Dzyuba, MD (The Hematology/Thrombosis Treatment Program, University Health Network, Toronto, Ontario, Canada), for her help with data collection and entry.

\section{REFERENCES}

1. Asmundson GJ, Norton PJ, Norton GR. Beyond pain: the role of fear and avoidance in chronicity. Clin Psychol Rev. 1999;19: 97-119.

2. Vlaeyen JW, Linton SJ. Fear-avoidance and its consequences in chronic musculoskeletal pain: a state of the art. Pain. 2000:85:317-332
3. Turk DC. A diathesis-stress model of chronic pain and disability following traumatic injury. Pain Res Manag. 2002;7: 9-19.

4. Reiss S. The expectancy model of fear, anxiety, and panic. Clin Psychol Rev. 1991;11:141-153.

5. Keogh E, Birkby J. The effect of anxiety sensitivity and gender on the experience of pain. Cogn Emot. 1999;13:813-829.

6. Asmundson GJ, Norton GR. Anxiety sensitivity in patients with physically unexplained chronic back pain: a preliminary report. Behav Res Ther. 1995;33:771-777.

7. Asmundson GJ, Taylor S. Role of anxiety sensitivity in painrelated fear and avoidance. J Behav Med. 1996;19:577-586

8. Asmundson GJ, Norton PJ, Veloso F. Anxiety sensitivity and fear of pain in patients with recurring headaches. Behav Med. 1999;19:577-586

9. Zvolensky MJ, Goodie JL, McNeil DW, et al. Anxiety sensitivity in the prediction of pain-related fear and anxiety in a heterogeneous chronic pain population. Behav Res Ther. 2001;39:683-696

10. Martin AL, McGrath PM, Brown SC, et al. Anxiety sensitivity, fear of pain and pain-related disability in children and adolescents with chronic pain. Pain Res Manag. 2007;12: 267-272.

11. McCracken LM, Gross RT, Sorg PJ, et al. Prediction of pain in patients with chronic low back pain: effects of inaccurate prediction and pain-related anxiety. Behav Res Ther. 1993;31: $647-652$.

12. Crombez $G$, Vervaet $L$, Lysens $R$, et al. Avoidance and confrontation of painful, back training movements in chronic back pain patients. Behav Modif. 1998:2:62-77.

13. Vlaeyen JWS, Kole-Snijders AMJ, Boeren RGB, et al. Fear of movement/(re)injury in chronic low back pain and its relation to behavioral performance. Pain. 1995;62:363-372.

14. Vlaeyen JWS, Kole-Sniders A, Rooteveel A, et al. The role of fear of movement/(re)injury in pain disability. J Occup Rehabil. $1995 ; 5: 235-252$

15. McCracken LM, Zayfert C, Gross RT. The pain anxiety symptoms scale: development and validation of a scale to measure fear of pain. Pain. 1992;67-73.

16. Quartana PJ, Campbell CM, Edwards RR. Pain catastrophizing: a critical review. Expert Rev Neurother. 2009;9:745-758.

17. Sullivan MJL, Bishop SR, Pivik J. The Pain Catastrophizing Scale: development and validation. Psychol Assess. 1995;7: 524-532.

18. Sullivan MJL, Thom B, Haythornthwaite JA, et al. Theoretical perspectives on the relation between catastrophizing and pain. Clin J Pain. 2001;17:52-64.

19. Keefe FJ, Brown GK, Wallston KA, et al. Coping with rheumatoid arthritis pain: catastrophizing as a maladaptive strategy. Pain. 1989;37:51-56.

20. Flor H, Behle DJ, Birbaumer N. Assessment of pain-related cognitions in pain patients. Behav Res Ther. 1993;31:63-73.

21. Sullivan MJL, D'Eon J. Relation between catastrophizing and depression in chronic pain patients. J Abnorm Psychol. 1990; 99:260-263

22. Sullivan MJL, Neish N. Catastrophizing, anxiety and pain during dental hygiene treatment. Community Dent Oral Epidemiol. 1998;37:243-250.

23. Jacobsen PB, Butler RW. Relation of cognitive coping and catastrophizing to acute pain and analgesic use following breast cancer surgery. J Behav Med. 1996;19:17-29.

24. Pavlin DJ, Sullivan MJ, Freund PR, et al. Catastrophizing: a risk factor for postsurgical pain. Clin J Pain. 2005;21: $83-90$.

25. Granot M, Ferber SG. The roles of pain catastrophizing and anxiety in the prediction of postoperative pain intensity: a prospective study. Clin J Pain. 2005;21:439-445.

26. Roth ML, Tripp DA, Harrison MH, et al. Demographic and psychosocial predictors of acute perioperative pain for total knee arthroplasty. Pain Res Manag. 2007;12:185-194.

27. Forsythe M, Dunbar M, Hennigar A, et al. Prospective relation between catastrophizing and residual pain following 
knee arthroplasty: two-year follow-up. Pain Res Manag. 2008; 13:335-341.

28. Sullivan M, Tanzer M, Stanish W, et al. Psychological determinants of problematic outcomes following total knee arthroplasty. Pain. 2009;143:123-129.

29. Philips HC. Avoidance behaviour and its role in sustaining chronic pain. Behav Res Ther. 1987;25:273-279.

30. Lethem J, Slade PD, Troup JD, et al. Outline of a fearavoidance model of exaggerated pain perception: I. Behav Res Ther. 1983;21:401-408.

31. Waddell G, Newton M, Henderson I, et al. A fear-avoidance beliefs questionnaire (FABQ) and the role of fear-avoidance beliefs in chronic low back pain and disability. Pain. 1993;52: $157-168$

32. Cook AJ, Brawer PA, Vowles KE. The fear-avoidance model of chronic pain: validation and age analysis using structural equation modeling. Pain. 2006;121:195-206.

33. Norton PJ, Asmundson GJG. Anxiety sensitivity, fear, and avoidance behavior in headache pain. Pain. 2004:111:218-223.

34. Asmundson GJG, Coons MJ, Taylor S, et al. PTSD and the experience of pain: research and clinical implications of shared vulnerability and mutual maintenance models. Can J Psychiatry. 2002;47:930-937.

35. Sharp TJ, Harvey AG. Chronic pain and posttraumatic stress disorder: mutual maintenance? Clin Psychol Rev. 2001;21: $857-877$.

36. Otis JD, Keane TM, Kerns RD. An examination of the relationship between chronic pain and post-traumatic stress disorder. J Rehabil Res Dev. 2003;40:397-406.

37. Pagé GM, Kleiman V, Asmundson GJ, et al. Structure of posttraumatic stress disorder symptoms in pain and pain-free patients scheduled for major surgery. J Pain. 2009;10:984-991.

38. Reiss S, Peterson RP, Gursky DM, et al. Anxiety sensitivity, anxiety frequency, and the prediction of fearfulness. Behav Res Ther. 1986;24:1-8.

39. McCracken LM, Dhingra L. A short version of the Pain Anxiety Symptoms Scale (PASS-20): preliminary development and validity. Pain Res Manag. 2002;7:45-50.

40. Pollard CA. Preliminary validity study of Pain Disability Index. Percept Mot Skills. 1984;59:974.

41. Weathers FW, Litz BT, Herman DS, et al. The PTSD Checklist-Civillian Version $(P C L-C)$. Available from F. W. Weathers, National Center for PTSD, Boston Veterans Affairs Medical Center, $150 \mathrm{~S}$. Huntington Avenue, Boston, MA 02130: 1991.

42. Zinbarg RE, Barlow DH, Brown TA. Hierarchical structure and general factor saturation of the Anxiety Sensitivity Index: evidence and implications. Psychol Assess. 1997;9:277-284.

43. Rodriguez BF, Bruce SE, Pagano ME, et al. Factor structure and stability of the Anxiety Sensitivity Index in a longitudinal study of anxiety disorder patients. Behav Res Ther. 2004;42: $79-91$

44. Osman A, Barrios FX, Gutierrez PM, et al. The Pain Catastrophizing Scale: further psychometric evaluation with adult samples. $J$ Behav Med. 2000;23:351-365.

45. Sullivan M, Bishop S, Pivik J. The pain catastrophizing scale: development and validation. Psychol Assess. 1995;7:524-532.

46. Abrams MP, Carleton RN, Asmundson GJG. An exploration of the psychometric properties of the PASS-20 with a nonclinical sample. J Pain. 2007;8:879-886.

47. Tait RC, Chibnall JT, Krause S. The Pain Disability Index: psychometric properties. Pain. 1990;40:171-182.

48. Jerome A, Gross RT. Pain Disability Index: construct and discriminant validity. Arch Phys Med Rehabil. 1991;72: 920-922.

49. Gronblad M, Hupli M, Wennerstrand P, et al. Intercorrelations and test-retest reliability of the Pain Disability Index (PDI) and the Oswestry Disability Questionnaire (ODQ) and their correlation with pain intensity in low back pain patients. Clin J Pain. 1993;9:189-195.
50. Tait RC, Pollard CA, Margolis RB, et al. The Pain Disability Index: psychometric and validity data. Arch Phys Med Rehabil. 1987;68:438-441

51. Asmundson GJG, Stapleton JA, Taylor S. Are avoidance and numbing distinct PTSD symptom clusters? J Trauma Stress. $2004 ; 17$.

52. Weathers FW, Litz BT, Herman DS, et al. The PTSD checklist: Reliability, validity, \& diagnostic utility. Paper presented at the Annual Meeting of the International Society for Traumatic Stress Studies; San Antonio Tx; 1993.

53. Blanchard EB, Jones-Alexander J, Buckley TC, et al. Psychometric properties of the PTSD Checklist (PCL). Behav Res Ther. 1996:34:669-673.

54. Katz J, Melzack R. Measurement of pain. Surg Clin North Am. 1999;79:231-252.

55. Bijur PE, Latimer CT, Gallagher EJ. Validation of a verbally administered numerical rating scale of acute pain for use in the emergency department. Emerg Med. 2003;10:390-392.

56. Williamson A, Hoggart B. Pain: a review of three commonly used pain rating scales. J Clin Nurs. 2001;14:789-804.

57. Coffman DL, MacCallum RC. Using parcels to convert path analysis models into latent variable models. Multivariate Behav Res. 2005; 40:235-259.

58. Breslau N, Kessler RC, Chilcoat HD, et al. Trauma and posttraumatic stress disorder in the community: the 1996 Detroit Area Survey of Trauma. Arch Gen Psychiatry. 1998;55:626-632.

59. American Psychiatric Association. Diagnostic and Statistical Manual for Mental Disorders. 4th ed. Text Revision. Washington, DC: American Psychiatric Association; 2000.

60. Mylle J, Maes M. Partial posttraumatic stress disorder revisited. J Affect Disord. 2004;78:37-48.

61. Arbuckle JL. Full information estimation in the presence of incomplete data. In: Marcoulides GA, Schumacker RE, eds. Advanced Structural Equation Modeling. Mahwah, NJ: Erlbaum; 1996;243-277.

62. Kline RB. Principles and Practice of Structural Equation Modeling. 2nd ed. New York: Guilford Press; 2005.

63. Hoyle RH, Panter AT. Writing about structural equation models. In: Hoyle RH, ed. Structural Equation Modeling: Concepts, Issues, and Applications. Thousand Oaks, CA: Sage Pulications; 1999:158-176.

64. Browne MW, Cudeck R. Single sample cross-validation indices for covariance structures. Multivariate Behav Res. 1989;24: 445-455.

65. Byrne BM. Structural Equation Modeling with Amos: Basic Concepts, Applications, and Programming. Mahwah, NJ: Lawrence Erlbaum Associates; 2001.

66. MacCallum RC. Working with imperfect models. Multivariate Behav Res. 2003;38:113-139.

67. MacCallum RC, Austin JT. Applications of structural equation modeling in psychological research. Anmu Rev Psychol. 2000;51:201-226.

68. Norton PJ, Asmundson GJG. Amending the fear-avoidance model of chronic pain: what is the role of physiological arousal? Behav Ther 2003;34:17-30.

69. Katz J, Asmundson GJG, McRae K, et al. Emotional numbing and pain intensity predict the development of pain disability up to one year after lateral thoracotomy. Eur J Pain. 2009; $13: 870-878$

70. Asmundson GJG, Bonin MF, Frombach IK, et al. Evidence of a disposition toward fearfulness and vulnerability to posttraumatic stress in dysfunctional pain patients. Behav Res Ther. 2000:38:801-812.

71. Asmundson GJ, Katz J. Understanding pain and posttraumatic stress disorder comorbidity: do pathological responses to trauma alter the perception of pain? Pain. 2008;31:247-249.

72. Ullman JB. Structural equation modeling. In: Tabachnick BE, Fidell LS, eds. Using Multivariate Statistics. 4th eds. Boston, MA: Pearson Education, Inc; 2001;653-771. 